\title{
Snarks with total chromatic number 5
}

\author{
Gunnar Brinkmann ${ }^{1} \quad$ Myriam Preissmann $^{2} \quad$ Diana Sasaki $\left.^{2}\right|^{*}$ \\ 1 Vakgroep Toegepaste Wiskunde en Informatica - Universiteit Gent, Belgium \\ 2 G-SCOP, Univ. Grenoble Alpes \& CNRS, France \\ 3 COPPE, Universidade Federal do Rio de Janeiro, Brazil
}

received $17^{\text {th }}$ Mar. 2014, revised $6^{\text {th }}$ May 2014, accepted $7^{\text {th }}$ May 2015.

A $k$-total-coloring of $G$ is an assignment of $k$ colors to the edges and vertices of $G$, so that adjacent and incident elements have different colors. The total chromatic number of $G$, denoted by $\chi_{T}(G)$, is the least $k$ for which $G$ has a $k$-total-coloring. It was proved by Rosenfeld that the total chromatic number of a cubic graph is either 4 or 5 . Cubic graphs with $\chi_{T}=4$ are said to be Type 1 , and cubic graphs with $\chi_{T}=5$ are said to be Type 2 .

Snarks are cyclically 4-edge-connected cubic graphs that do not allow a 3-edge-coloring. In 2003, Cavicchioli et al. asked for a Type 2 snark with girth at least 5 . As neither Type 2 cubic graphs with girth at least 5 nor Type 2 snarks are known, this is taking two steps at once, and the two requirements of being a snark and having girth at least 5 should better be treated independently.

In this paper we will show that the property of being a snark can be combined with being Type 2 . We will give a construction that gives Type 2 snarks for each even vertex number $n \geq 40$.

We will also give the result of a computer search showing that among all Type 2 cubic graphs on up to 32 vertices, all but three contain an induced chordless cycle of length 4 . These three exceptions contain triangles. The question of the existence of a Type 2 cubic graph with girth at least 5 remains open.

Keywords: snark, total-coloring, edge-coloring

\section{Introduction}

In 1880, Tait [27] proved that the Four-Color Conjecture is equivalent to the statement that every planar bridgeless cubic graph has chromatic index 3 . The search for counterexamples to the Four-Color Conjecture motivated the study of cubic graphs with chromatic index 4. Based on the poem by Lewis Carroll "The Hunting of the Snark", Gardner [16] introduced the name snark for nontrivial cubic graphs that are not 3-edge-colorable, without giving a precise definition for "nontrivial".

The importance of these graphs arises also from the fact that several well known conjectures would have snarks as minimal counterexamples [10], among them Tutte's 5-Flow Conjecture [28], the 1-Factor Double Cover Conjecture [15], and the Cycle Double Cover Conjecture [24, 26]. In [5] snarks prove their potential as counterexamples by refuting 8 published conjectures.

\footnotetext{
* Supported by CAPES/COFECUB and CNPq. 
The Petersen graph is the smallest and earliest known snark. It is known that there are no snarks of order 12,14 or 16 (see for example [13, 14]). In [19] Isaacs introduced the dot product, a famous operation used for constructing infinitely many snarks, and defined the Flower snark family. The Blanuša snark [2] of order 18 is constructed using the dot product of two copies of the Petersen graph, and Preissmann [21] proved that there are only two snarks of order 18. There are snarks of any even order bigger than 18 .

In [9] Cavicchioli et al. reported that their extensive computer study of snarks shows that all snarks of girth at least 5 and with less than 30 vertices are Type 1, and asked for the smallest order of a snark of girth at least 5 that is Type 2. Later on Brinkmann et al. [5] have shown that this order should be at least 38. In 2011, it was proved that all members of the infinite families of Flower and Goldberg snarks are Type $1[8]$. Recently, it has been proved that all members of another two infinite families of snarks are Type 1 [11].

The question of Cavicchioli et al. has two requirements for the Type 2 cubic graphs and so far for none of these two requirements a Type 2 cubic graph is known. Therefore one should split the question in two parts in order to investigate what the real obstruction is that makes these graphs so hard to find (if they exist at all): being snarks or having girth at least 5 .

- Does there exist a Type 2 cubic graph with girth at least 5 ?

- Does there exist a Type 2 snark?

In this paper we will give a positive answer to the last question and a construction that provides an infinite sequence of Type 2 snarks. The first question remains unsolved, but our computational results show that a possible Type 2 cubic graph with girth at least 5 must have at least 34 vertices. Furthermore it seems that, even for girth 3, Type 2 cubic graphs with no induced chordless cycles of length 4 are difficult to find.

\section{Definitions}

In this section, we give some definitions and notation that will be used in this paper.

A semi-graph is a 3-tuple $G=(V, E, S)$ where $V$ is the set of vertices of $G, E$ is a set of edges having two distinct endpoints in $V$, and $S$ is a set of semi-edges having one endpoint in $V$.

We will write $e=v w$ or $s=v$, though the endpoints of an edge or semi-edge do not determine it uniquely. When vertex $v$ is an endpoint of $e \in E \cup S$ we will say that $v$ and $e$ are incident. Two elements of $E \cup S$ incident to the same vertex, respectively two vertices incident to the same edge, will be called adjacent.

A graph $G$ is a semi-graph with an empty set of semi-edges. In that case we can write $G=(V, E)$. Given a semi-graph $G=(V, E, S)$, we call the graph $(V, E)$ the underlying graph of $G$. Notice that we can also associate to $G$ the graph $G^{\prime}$ obtained from $G$ as follows: for each semi-edge $s=v$. of $G$, create a new vertex $x_{s}$ and replace $s$ by a new edge $v x_{s}$.

All definitions given below for semi-graphs, that do not require the existence of semi-edges, are also valid for graphs.

Let $G=(V, E, S)$ be a semi-graph.

The degree $d(v)$ of a vertex $v$ of $G$ is the number of elements of $E \cup S$ that are incident to $v$. We say that $G$ is $d$-regular if the degree of each vertex is equal to $d$. In this paper we are mainly interested in 3-regular graphs and semi-graphs, also called respectively cubic graphs and cubic semi-graphs. Given 
a graph $G$ of maximum degree 3, the semi-graph obtained from $G$ by adding $3-d(v)$ semi-edges with endpoint $v$, for each vertex $v$ of $G$, is called the cubic semi-graph generated by $G$ and is denoted by $s-G$.

For $k \in \mathbb{N}$, a $k$-vertex-coloring of $G$ is a map $C^{V}: V \rightarrow\{1,2, \ldots, k\}$, such that $C^{V}(x) \neq C^{V}(y)$ whenever $x$ and $y$ are two adjacent vertices. The chromatic number of $G$, denoted by $\chi(G)$, is the least $k$ for which $G$ has a $k$-vertex-coloring.

Similarly, a $k$-edge-coloring of $G$ is a map $C: E \cup S \rightarrow\{1,2, \ldots, k\}$, such that $C(e) \neq C(f)$ whenever $e$ and $f$ are adjacent elements of $E \cup S$. The chromatic index of $\mathrm{G}$, denoted by $\chi^{\prime}(G)$, is the least $k$ for which $G$ has a $k$-edge-coloring. By Vizing's theorem [29] we have that $\chi^{\prime}(G)$ is equal to either $\Delta(G)$ or to $\Delta(G)+1$, where $\Delta(G)$ is the maximum degree of the vertices of $G$. If $\chi^{\prime}(G)=\Delta(G)$, then $G$ is said to be Class 1, otherwise $G$ is said to be Class 2 .

A $k$-total-coloring of $G$ is a map $C^{T}: V \cup E \cup S \rightarrow\{1,2, \ldots, k\}$, such that

(a) $\left.C^{T}\right|_{V}$ is a vertex-coloring,

(b) $\left.C^{T}\right|_{E \cup S}$ is an edge-coloring,

(c) $C^{T}(e) \neq C^{T}(v)$ whenever $e \in E \cup S, v \in V$, and $e$ is incident to $v$.

The total chromatic number of $G$, denoted by $\chi_{T}(G)$, is the least $k$ for which $G$ has a $k$-total-coloring. Clearly $\chi_{T}(G) \geq \Delta(G)+1$. The Total Coloring Conjecture [1, 29] claims that $\chi_{T}(G) \leq \Delta+2$. If $\chi_{T}(G)=\Delta(G)+1$, then $G$ is said to be Type 1, and if $\chi_{T}(G)=\Delta(G)+2$, then $G$ is said to be Type 2 .

The Total Coloring Conjecture has been proved for cubic graphs [23]. Thus, a cubic graph is either Type 1 (its total chromatic number is equal to 4 ) or Type 2 (its total chromatic number is equal to 5).

We will show now that $(k+1)$-total-colorings of $k$-regular semi-graphs are characterized by some particular $(k+1)$-edge-colorings, so that in a later proof we do not have to care about vertex-coloring.

Definition $1 A(k+1)$-edge-coloring $C$ of a $k$-regular semi-graph $G=(V, E, S)$ is called a strong $(k+1)$-edge-coloring if for each edge $v w \in E$ we have

$\mid\{C(e) \mid e \in E \cup S, e$ incident to $v$ or $w\} \mid=k+1$.

Equivalently a strong $(k+1)$-edge-coloring of a $k$-regular semi-graph is a $(k+1)$-edge-coloring such that for each edge $v w$ the color not used for the elements of $E \cup S$ incident to $v$ must be used for an element incident to $w$. A strong $(k+1)$-edge-coloring has the property that it is not possible to obtain another $(k+1)$-edge-coloring by changing the color of a single edge.

Lemma 1 Let $G=(V, E, S)$ be a k-regular semi-graph.

Each strong $(k+1)$-edge-coloring $C$ of $G$ can be extended to a $(k+1)$-total-coloring $C^{T}$ with $\left.C^{T}\right|_{E \cup S}=C$, and, for each $(k+1)$-total-coloring $C^{T}$ of $G,\left.C^{T}\right|_{E \cup S}$ is a strong $(k+1)$-edge-coloring.

This implies:

There exists $a(k+1)$-total-coloring $C^{T}$ of $G$ if and only if there exists a strong $(k+1)$-edge-coloring $C$ of $G$.

Proof: If $C$ is a $(k+1)$-edge-coloring of $G$ then, for any vertex $v$, all $k$ elements of $E \cup S$ incident to $v$ are colored differently. So, there exists a $(k+1)$-total-coloring $C^{T}$ of $G$ such that $C=\left.C^{T}\right|_{E \cup S}$ if and only if the function $C^{V}: V \rightarrow\{1,2, \ldots, k+1\}$, such that $C^{V}(v)$ is equal to the unique color not used by $C$ for the elements of $E \cup S$ incident to $v$, is a $(k+1)$-vertex-coloring of $G$ (thus extending $C$ to a total coloring). 
By Lemma 1, a cubic semi-graph $G$ is of Type 1 if and only if there exists a strong 4-edge-coloring of $G$. This will be used later to prove that some semi-graphs are not of Type 1 and in a computer program computing the type of cubic graphs.

The following Lemma is well known and useful.

Lemma 2 Parity Lemma (Blanuša, 1946 [2] - Descartes, 1948 [12]) Let G be a cubic semi-graph containing exactly $k$ semi-edges, $C$ be a 3 -edge-coloring of $G$, and $k_{1}, k_{2}, k_{3}$ be the number of semi-edges of $G$ colored respectively $1,2,3$ by $C$. Then

$$
k_{1} \equiv k_{2} \equiv k_{3} \equiv k \bmod (2) .
$$

A triangle is a graph consisting of a cycle of length 3 (or equivalently a complete graph on three vertices), a square is a graph consisting of a chordless cycle of length 4 , and an s-square is a cubic semigraph generated by a square. A $K_{4}$ is a complete graph on four vertices.

In the rest of this section, $G=(V, E)$ will be assumed to be a graph.

The girth of $G$ is the minimum length of a cycle contained in $G$, or if $G$ has no cycle, it is defined to be infinity. A subgraph of $G$ is any graph $G^{\prime}=\left(V^{\prime}, E^{\prime}\right)$ such that $V^{\prime} \subseteq V$ and $E^{\prime} \subseteq E$. An induced subgraph of $G$ is any subgraph $G^{\prime}=\left(V^{\prime}, E^{\prime}\right)$ such that $E^{\prime}$ is equal to the set of edges of $G$ with both endpoints in $V^{\prime}$. We will denote it by $G\left[V^{\prime}\right]$. Given a graph $H, G$ will be called $H$-free if none of its induced subgraphs are isomorphic to $H$.

Let $A$ be a proper subset of $V$. We call the set $F$ of edges of $G$ with one endpoint in $A$ and the other endpoint in $V \backslash A$, the edge cutset induced by $A$. A subset $F$ of edges of $\mathrm{G}$ is an edge cutset if there exists a proper subset $A$ of $V$ such that $F$ is the edge cutset induced by $A$. If none of $G[A]$ and $G[V \backslash A]$ is acyclic, then $F$ is said to be a c-cutset.

We say that $G$ is cyclically k-edge-connected if it has not c-cutset of cardinality smaller than $k$. If $G$ has at least one c-cutset, the cyclic-edge-connectivity of $G$ is the smallest cardinality of a c-cutset of $G$, else it is set to infinity. It is useful to notice the following.

Remark 1 A cubic graph is cyclically 4-edge-connected if and only if each of its edge cutsets of cardinality smaller than 4 is induced by a single vertex.

In this paper, a snark is a cyclically 4-edge-connected Class 2 cubic graph. Some authors use other definitions [25, 30], but most commonly, snarks are defined as cyclically 4-edge-connected Class 2 cubic graphs, with sometimes the additional property to be square-free. The name "snark" was proposed as a substitute for "Nontrivial trivalent graphs which are not Tait-colorable" used by Isaacs [19]. With respect to a cubic graph being Type 2, neither a square nor a small cyclic-edge-connectivity seem to be "trivial" like it is the case for being Class 2 (see the graphs in [11] and [18]).

\subsection{The principle of the construction}

The idea of our construction of Type 2 snarks is based on the following fact.

Remark 2 A graph $G$ containing a Class 2 (resp. Type 2) subgraph with maximum degree $\Delta(G)$ is Class 2 (resp. Type 2). 
So a way to build a Type 2 snark is to make a cyclically 4-edge-connected cubic graph from a graph of maximum degree 3 which is Class 2 and a graph of maximum degree 3 which is Type 2 . This is what is explained in this subsection.

Definition $2 A$ brick is a cubic semi-graph $B=(V, E, S)$ with exactly four pairwise non-adjacent semiedges and whose underlying graph $(V, E)$ is a subgraph of some cyclically 4-edge-connected cubic graph.

Given two disjoint cubic semi-graphs with exactly four pairwise non-adjacent semi-edges, $B=(V, E, S)$ and $B^{\prime}=\left(V^{\prime}, E^{\prime}, S^{\prime}\right)$, any graph $G=\left(V \cup V^{\prime}, E \cup E^{\prime} \cup E^{\prime \prime}\right)$ with $E^{\prime \prime}$ being a set of four disjoint edges $x y$ with $x \cdot \in S$ and $y \cdot \in S^{\prime}$, is called a junction of $B$ and $B^{\prime}$.

Lemma 3 (a) The underlying graph of a brick is bridgeless.

(b) Any junction of two bricks is a cyclically 4-edge-connected graph.

(c) An s-square is a brick. No brick has less than four vertices.

(d) Let B be a cubic semi-graph with exactly four pairwise non-adjacent semi-edges. The three following propositions are equivalent:

1) $B$ is a brick,

2) any junction of $B$ and an s-square is cyclically 4-edge-connected,

3) there is a junction of $B$ with an s-square that is cyclically 4-edge-connected.

Proof: (a) Let $G_{B}$ be the underlying graph of a brick $B$. Then $G_{B}$ has four vertices of degree 2 and all others have degree 3 . Since $B$ is a brick, $G_{B}$ is the subgraph of some cyclically 4-edge-connected cubic graph $H$.

Suppose to the contrary that $G_{B}$ has a bridge. The fact that $G_{B}$ has minimum degree 2 implies that after the removal of the bridge both components contain a cycle. At least one of the components contains at most two vertices of degree $2-$ in $H$ this component is attached to the rest by at most three edges - so $H$ has a c-cutset of size at most 3 - a contradiction.

(b) Let $B=(V, E, S)$ and $B^{\prime}=\left(V^{\prime}, E^{\prime}, S^{\prime}\right)$ be disjoint bricks and $G$ be any graph obtained by the junction of $B$ and $B^{\prime}$. If $G$ would contain a c-cutset of cardinality at most 3 (possibly with edges that are neither in $B$ nor in $B^{\prime}$ ), then one of $B, B^{\prime}$ would contain at least two edges of this c-cutset, because otherwise, by (a), both $B$ and $B^{\prime}$ would be connected after removing the edges, and also connected to each other by at least one edge. So assume that $B$ contains at least two edges of the c-cutset, implying that $B^{\prime}$ contains at most one. But then, as $B^{\prime}$ stays connected by (a), one of the cyclic components after removal of the c-cutset must be entirely in $B$ - so this component could be split off by removing at most three edges in any cubic graph containing the underlying graph of $B-$ a contradiction.

(c) The proof is immediate as there are cyclically 4-edge-connected cubic graphs that have squares as subgraphs, as for example $K_{4}$ or the 3-dimensional cube. Furthermore, by definition a brick should have at least four vertices.

(d) $[1 \Rightarrow 2]$ Let us assume that $B$ is a brick. By (c), an $s$-square is also a brick, and by (b) we get that any junction of $B$ and an $s$-square is cyclically 4-edge-connected.

[2 $\Rightarrow 3$ ] Assuming 2), it is enough to prove that there exists a junction of $B$ and an $s$-square, which is immediate. 
[3 $\Rightarrow 1$ ] Let $G$ be a cyclically 4-edge-connected graph obtained from a junction of $B$ and an $s$-square. The underlying graph of $B$ is a subgraph of $G$. So with our hypothesis on $B$ we get that $B$ is a brick.

Theorem 1 A graph obtained by a junction of a Class 2 brick and a Type 2 brick is a Type 2 snark.

Proof: Immediate from Lemma 3 and Remark 2

So we are now lead to the problem of finding a Class 2 brick and a Type 2 brick.

\section{Finding Class 2 bricks}

In this section, we will show how to obtain Class 2 bricks and prove that the smallest ones have 18 vertices.

Definition 3 Let $G$ be a cyclically 4-edge-connected cubic graph.

Removing two non-adjacent edges of $G$, one obtains a graph generating a cubic semi-graph $B$ with exactly four pairwise non-adjacent semi-edges, which is by definition a brick. Such a brick is called a direct-brick of $G$. Two non-adjacent vertices of a direct-brick $B$ of $G$ that are adjacent in $G$ are called $a$ pair of $B$ (with respect to $G$ ). Two semi-edges incident to vertices of the same pair of $B$ are also called $a$ pair. By definition a direct-brick of $G$ contains two pairs of semi-edges.

Removing two adjacent vertices of $G$ and all their incident edges, if $G$ has at least six vertices, one obtains a graph generating a cubic semi-graph $B$ with exactly four pairwise non-adjacent semi-edges, which is by definition a brick. Such a brick is called an edge-brick of $G$. Two vertices of $B$ that are in $G$ adjacent to the same vertex not in $B$ are called a pair of $B$ (with respect to $G$ ). Two semi-edges incident to vertices of the same pair of $B$ are also called a pair. By definition an edge-brick of $G$ contains two pairs of semi-edges.

Notice that there exist bricks that are not direct-bricks. One example can be downloaded from the graph database HoG [6] by searching for the keyword Not_direct_brick [7]. On another hand, we could show that any brick is an edge-brick of some cyclically 4-edge-connected cubic graph [7].

We will show that Class 2 bricks can be obtained from the dot product of snarks. Before explaining this, we will give some useful properties that were noticed by Isaacs.

Lemma 4 (Isaacs, 1975 [19]) Let $G$ be a snark and let $B$ be a direct-brick of $G$. In every 3-edge-coloring of $B$, two semi-edges that form a pair with respect to $G$ get distinct colors.

Lemma 5 (Isaacs, 1975 [19]) Let $G$ be a snark and let B be an edge-brick of $G$. In every 3-edge-coloring of $B$, the semi-edges that are in a pair with respect to $G$ get the same color.

Lemmas 4 and 5 follow immediately from the Parity Lemma and the fact that a snark is a Class 2 graph.

Definition 4 Let $S_{1}, S_{2}$ be disjoint snarks, $B_{1}=\left(V_{1}, E_{1}, S_{1}\right)$ a direct-brick of $S_{1}$ with vertex pairs $r_{1}, r_{2}$ and $s_{1}, s_{2}$ and $B_{2}=\left(V_{2}, E_{2}, S_{2}\right)$ an edge-brick of $S_{2}$ with vertex pairs $x_{1}, x_{2}$ and $y_{1}, y_{2}$.

Then $D=\left(V_{1} \cup V_{2}, E_{1} \cup E_{2} \cup\left\{r_{1} x_{1}, r_{2} x_{2}, s_{1} y_{1}, s_{2} y_{2}\right\}\right)$ is called a dot product of $S_{1}, S_{2}$.

So, a dot product is a pair-to-pair junction of one direct-brick and one edge-brick, both bricks arising from snarks. The dot product is depicted in Figure 1.

The following theorem is an easy consequence of Lemmas 4 and 5

Theorem 2 (Isaacs, 1975 [19]) Any dot product of two snarks is a snark. 

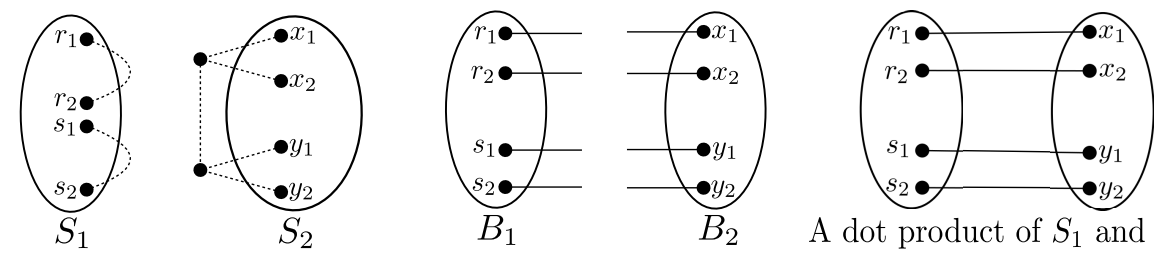

A dot product of $S_{1}$ and $S_{2}$

Fig. 1: An illustration of the dot product of $S_{1}$ and $S_{2}$.

Definition 5 Let $S_{1}, S_{2}$ be two disjoint snarks, $B_{1}$ be a direct-brick of $S_{1}$ with vertex pairs $r_{1}, r_{2}$ and $s_{1}, s_{2}$ and $B_{2}$ be an edge-brick of $S_{2}$ with vertex pairs $x_{1}, x_{2}$ and $y_{1}, y_{2}$.

Furthermore, let $D=(V, E)$ be the dot product of $S_{1}, S_{2}$ with edges

$\left\{r_{1} x_{1}, r_{2} x_{2}, s_{1} y_{1}, s_{2} y_{2}\right\}$ and $z$ a neighbor of $r_{2}$ in $D$.

Then $Z=\left(V, E^{\prime}, S^{\prime}\right)$ with $E^{\prime}=E \backslash\left\{r_{1} x_{1}, r_{2} z\right\}$ and $S^{\prime}=\left\{r_{1} \cdot, x_{1} \cdot, r_{2} \cdot, z \cdot\right\}$ is called a semi-dot product of $S_{1}, S_{2}$.

Lemma 6 A semi-dot product $Z$ of two snarks is a Class 2 brick.

Proof: By definition $Z$ is a direct-brick of a dot product of two snarks. What remains to be shown is that it is Class 2. We use the notation as in Definition 5

If $z=x_{2}$ the result follows exactly like in the proof of Theorem 2 as the contradiction in that proof already follows from the connections between one of the two sets of paired vertices.

So assume that $z \neq x_{2}$ and that $C$ is a 3-edge-coloring of $Z$. $C$ induces a 3-edge-coloring of $B_{2}$, so by Lemma 5. $C\left(r_{2} x_{2}\right)=C\left(x_{1} \cdot\right)$ which is w.l.o.g. equal to 1 . Then we can fix $C\left(r_{2} \cdot\right)=2$ and the Parity Lemma and Lemma 4 applied to $Z$ give $C\left(r_{1} \cdot\right)=2$ and $C(z \cdot)=1$.

But then $C^{\prime}$ defined as $C^{\prime}=C$ on edges of $B_{1}$ distinct from $r_{2} z, C^{\prime}\left(r_{2} z\right)=1, C^{\prime}\left(s_{1} \cdot\right)=C^{\prime}\left(s_{2} \cdot\right)=$ $C\left(s_{1} y_{1}\right)$ and $C^{\prime}\left(r_{1} \cdot\right)=C^{\prime}\left(r_{2} \cdot\right)=2$ is a 3-edge-coloring of $B_{1}$ contradicting Lemma 4

Theorem 3 The smallest Class 2 bricks have 18 vertices and there are 5 of them.

Proof: The Petersen graph is the smallest snark, so the smallest Class 2 bricks that can be obtained by the semi-dot product come from an edge-brick and a direct brick of the Petersen graph and have 18 vertices. Due to the high symmetry of the Petersen graph, there are only two direct-bricks and one edge-brick of the Petersen graph. Applying it in every possible way, the construction gives 5 non-isomorphic Class 2 bricks of order 18.

Using a computer and exhaustively enumerating all cubic semi-graphs with exactly four pairwise nonadjacent semi-edges up to 18 vertices and filtering them for Class 2 bricks, we checked that these are in fact the smallest Class 2 bricks and that they are all with 18 vertices. Figure 2 shows one of these graphs.

Note that some direct-bricks of Loupekhine snarks [20] can also be shown to be Class 2. The smallest such brick is the critical Class 2 graph of order 22 with maximum degree 3 due to Goldberg [17]. 


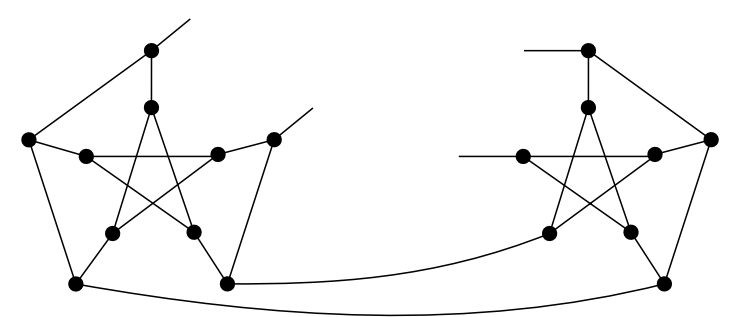

Fig. 2: One of the smallest Class 2 bricks.

\section{Finding Type 2 bricks}

Type 2 bricks were not easy to find. From Hamilton and Hilton [18] it was known that a Type 2 brick would have at least 18 vertices. A computer search determined that the smallest Type 2 brick has 22 vertices. It is depicted in Figure 3 and we will denote it $B^{*}$. We find it worth explaining why this graph is a brick of Type 2 .

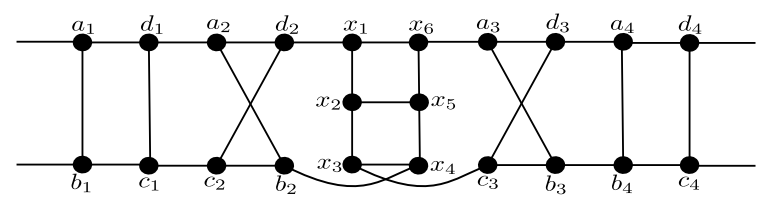

Fig. 3: The Type 2 brick $B^{*}$.

Lemma 7 In every 4-total-coloring of an s-square, semi-edges incident to adjacent vertices get distinct colors.

Proof: Let us consider an s-square $S$ with edges $a b, b c, c d, d a$ (Figure 4). Assume that the lemma is not true. Then there exists a 4-total-coloring $C^{T}$ of $S$ such that $a \cdot$ and $b$ - are colored the same. By Lemma 1 applied to edge $a b$, we can assume w.l.o.g. that $C^{T}(a \cdot)=1, C^{T}(b \cdot)=1, C^{T}(a b)=2, C^{T}(a d)=3$, and $C^{T}(b c)=4$. Moreover, applying Lemma 1 to edges $a d$ and $b c$, we get $C^{T}(d \cdot)=4$ and $C^{T}(c \cdot)=3$. But then, edge $c d$ cannot satisfy Lemma 1 , and we get a contradiction.

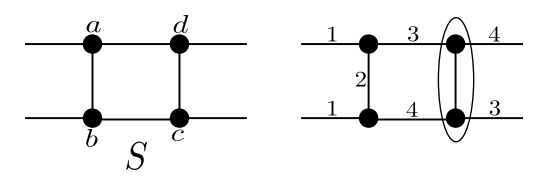

Fig. 4: An attempt to find a strong-edge-coloring of $S$.

Let us call the graph consisting of two squares sharing an edge, a domino, and the cubic semi-graph generated by a domino an s-domino. Two vertices at maximum distance in an s-domino are called opposite. An s-domino contains two pairs of opposite vertices. 
Lemma 8 Every 4-total-coloring $C^{T}$ of an s-domino is such that, for one pair of opposite vertices $x$ and $y$, the following holds: $C^{T}(x)=C^{T}(y)$ and $C^{T}(x \cdot) \neq C^{T}(y \cdot)$.

Proof: Let us consider an s-domino $D$ with edges $x_{1} x_{2}, x_{2} x_{3}, x_{3} x_{4}, x_{4} x_{5}, x_{5} x_{6}, x_{6} x_{1}, x_{2} x_{5}$, and let $C^{T}$ be a 4-total-coloring of $D$ (Figure 5).

By symmetry, by Lemma 1 for edge $x_{2} x_{5}$, and Lemma 7 , we can assume w.l.o.g. that $C^{T}\left(x_{2} x_{5}\right)=1$, $C^{T}\left(x_{1} x_{2}\right)=C^{T}\left(x_{5} x_{4}\right)=2, C^{T}\left(x_{2} x_{3}\right)=3$, and $C^{T}\left(x_{5} x_{6}\right)=4$.
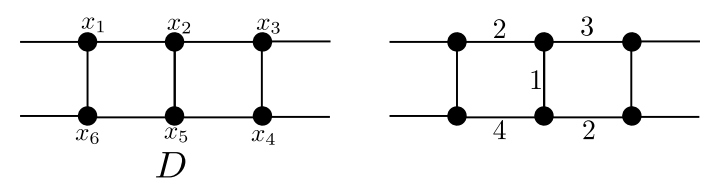

Fig. 5: Strong-edge-coloring applied to edge $x_{2} x_{5}$ of $D$.

By Lemma 1 for edge $x_{1} x_{2}$, and the fact that $C^{T}\left(x_{5} x_{6}\right)=4$, we have that $C^{T}\left(x_{1} \cdot\right)=4$; similarly, we must have $C^{T}\left(x_{4} \cdot\right)=3$. Lemma 1 for edge $x_{1} x_{6}$ implies that edge $x_{1} x_{6}$ and semi-edge $\left(x_{6} \cdot\right)$ must have colors 1 and 3 (not necessarily in this order). Similarly, Lemma 1 for edge $x_{3} x_{4}$ implies that edge $x_{3} x_{4}$ and semi-edge $\left(x_{3} \cdot\right)$ must have colors 1 and 4 .

In any case, $x_{6}$ and $x_{3}$ receive color 2 , so these vertices have the desired property except for the case where $C^{T}\left(x_{6} \cdot\right)=C^{T}\left(x_{3} \cdot\right)=1$. But in this case, we have $C^{T}\left(x_{3} x_{4}\right)=4$ and $C^{T}\left(x_{5} x_{6}\right)=3$, and so vertices $x_{1}$ and $x_{4}$ have the desired property.

Theorem 4 The semi-graph $B^{*}$ of Figure 3 is a brick of Type 2.

Proof: Consider the semi-graph $B^{*}$ in Figure 3 it is a cubic semi-graph with exactly four pairwise non-adjacent semi-edges and adding e.g. the non-adjacent edges $a_{1} d_{4}$ and $b_{1} c_{4}$ we obtain a cyclically 4-edge-connected cubic graph, so $B^{*}$ is a (direct-) brick. Now, we will prove that it is of Type 2.

Let us remark that vertices $x_{1}, x_{2}, x_{3}, x_{4}, x_{5}$, and $x_{6}$ of $B^{*}$ induce a domino in $B^{*}$. Furthermore, the opposite pairs of this domino are connected similarly to the same graph ( $B^{*}\left[a_{1}, b_{1}, c_{1}, d_{1}, a_{2}, b_{2}, c_{2}, d_{2}\right]$ is isomorphic to $\left.B^{*}\left[a_{3}, b_{3}, c_{3}, d_{3}, a_{4}, b_{4}, c_{4}, d_{4}\right]\right)$.

Assume that there exists a 4-total-coloring $C^{T}$ of $B^{*}$. Then, by Lemma 8 and the symmetry of $B^{*}$, one can assume w.l.o.g. that $C^{T}\left(x_{1}\right)=C^{T}\left(x_{4}\right)=1, C^{T}\left(x_{1} d_{2}\right)=2$, and $C^{T}\left(x_{4} b_{2}\right)=3$.

Now, by Lemma 7 applied to the s-square generated by $a_{2}, b_{2}, c_{2}, d_{2}$, edges $d_{1} a_{2}$ and $c_{1} c_{2}$ cannot be colored with colors 2 or 3 by $C^{T}$. On the other hand, by Lemma 7 applied to the s-square generated by $a_{1}, b_{1}, c_{1}, d_{1}$, edges $d_{1} a_{2}$ and $c_{1} c_{2}$ cannot be colored the same by $C^{T}$.

So, we can assume w.l.o.g. that $C^{T}\left(d_{1} a_{2}\right)=1$ and $C^{T}\left(c_{1} c_{2}\right)=4$.

Now, remark that since vertices $x_{1}$ and $x_{4}$ are colored 1 , there is no edge incident to them that is colored 1. So, by Lemma 1 applied to edges $x_{1} d_{2}$ and $x_{4} b_{2}$ and the fact edge $d_{1} a_{2}$ is colored 1 , we have that $C^{T}\left(d_{2} c_{2}\right)=C^{T}\left(b_{2} c_{2}\right)=1$, which is impossible. So, we get a contradiction and there is no 4-total-coloring of $B^{*}$.

Of course any brick that has the underlying graph of $B^{*}$ as a subgraph is Type 2. A computer search gave that up to 26 vertices this is the only way to obtain larger Type 2 bricks: 
Remark 3 There is one Type 2 brick with 22 vertices, it is the brick $B^{*}$ depicted in Figure 3 . There are two Type 2 bricks with 24 vertices and there are ten Type 2 bricks with 26 vertices. All these bricks contain the underlying graph of $B^{*}$ as a subgraph.

The 22 vertex brick can be downloaded from the graph database HoG [6] by searching for the keyword Type2_brick_22.

\section{Type 2 snarks}

Now, we are able to present the main result of this paper. Since a junction of a Class 2 brick with a Type 2 brick gives a Type 2 snark, we can construct Type 2 snarks.

Theorem 5 There exist Type 2 snarks for each even order $n \geq 40$.

Proof: Any junction of the Type 2 brick of order 22 of Figure 3 and a Class 2 brick of order 18 as described in Section 3 (Figure 2) is a Type 2 snark of order 40. Using a computer to construct all possible combinations it was found that up to isomorphism there are 11 such Type 2 snarks of order 40. Figure 6 presents one of them. All graphs can be downloaded from the graph database HoG [6] by searching for the keyword Type2_Class2_40.

From any Class 2 or Type 2 brick $B$ it is easy to obtain a new one with two more vertices $v$ and $w$ : delete two semi-edges $x \cdot, y$. and add edges $x v, v w, w y$ and semi-edges $v \cdot, w \cdot$. Indeed, the so obtained semi-graph is an edge-brick of the cyclically 4-edge-connected graph obtained by the junction of $B$ and a square; and it contains the underlying graph of $B$ as a subgraph. So there exist snarks of Type 2 for all even orders from 40 on.

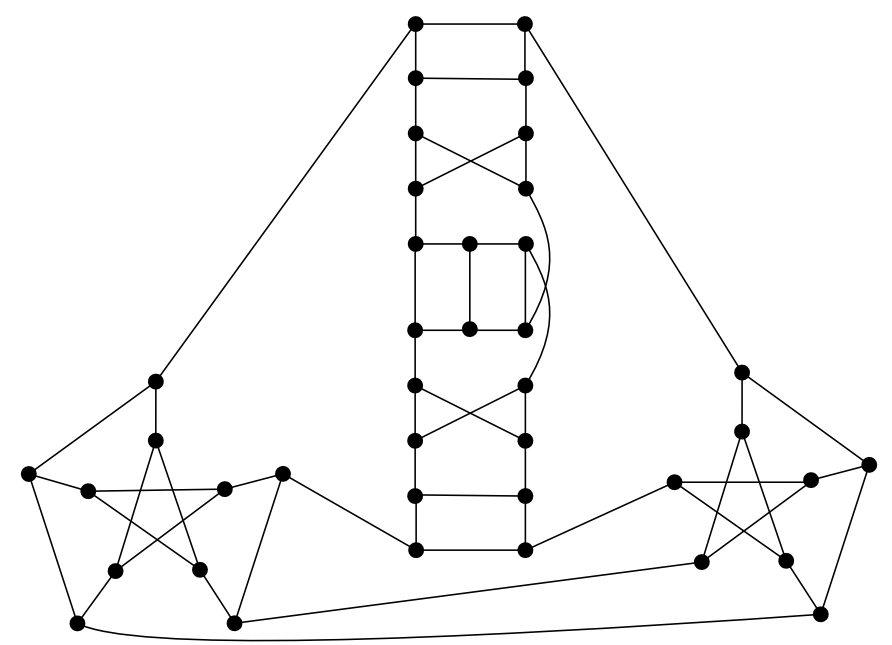

Fig. 6: One of the 11 smallest snarks of Type 2 obtained by a junction of the bricks on Figure 2 and Figure 3

Question 1 Does there exist a Type 2 snark of order less than 40? 
A computer search gave that all snarks on up to 34 vertices are Type 1, so the only possible orders for which the existence is not yet known are 36 and 38.

\section{Checking the programs}

For the generation of the cubic graphs, the program minibaum described in [3] was used. The snarks were generated by the program snarkhunter of which the construction and isomorphism routines are described in [4]. The cubic semi-graphs with exactly four pairwise non-adjacent semi-edges were generated by the program multigraph that is based on the techniques of minibaum. It was never published, but is used since almost 20 years and during this time the results were compared against the results of various other programs and there was never any discrepancy.

Except for the program testing the property of being a brick, most testing programs are also used for a long time. They were e.g. also used in [5] where most of the results were compared against independent results. For the largest cases (e.g. square-free cubic graphs on 32 vertices) where the total chromatic number had to be determined, a special program had to be developed. It uses Lemma 1 and searches for strong 4-edge-colorings. For testing purposes the results were compared to the results of the older, well tested and independent program for all cubic graphs up to 24 vertices and for triangle-free cubic graphs on 26 vertices and were found to be in complete agreement.

For testing whether a cubic semi-graph with exactly four pairwise non-adjacent semi-edges is a brick, two programs were written. One checked for cuts and the distribution of semi-edges in the components after removing the cuts, and the other (faster one) used Lemma 3 It did a junction of the cubic semigraph with four pairwise non-adjacent semi-edges with an s-square and tested the resulting graph for being cyclically 4-edge-connected. The programs were compared for all cubic semi-graphs with four pairwise non-adjacent semi-edges on up to 18 vertices (more than 4.000 .000 graphs) and were found to be in complete agreement.

\section{Conclusion}

In 2003, Cavicchioli et al. [9] reported that their extensive computer study of snarks shows that all snarks of girth at least 5 and with less than 30 vertices are Type 1, and asked for the smallest order of a snark of girth at least 5 that is Type 2. Later on Brinkmann et al. [5] have shown that this order should be at least 38 .

Relaxing the cyclic-edge-connectivity conditions, it is easy to find examples of Class 2 Type 2 cubic graphs of cyclic edge-connectivity less than 4 . In this paper we presented the first known Type 2 snarks. They are all of girth 4, as well as all known Type 2 cyclically 4-edge-connected cubic graphs distinct from $K_{4}$. More generally, it seems that no Type 2 cubic graph with girth greater than 4 is known. Therefore, we also propose the following question.

Question 2 What is the smallest Type 2 cubic graph with girth at least 5?

A computer search gave that all cubic graphs with girth 5 and up to 32 vertices are Type 1, so a smallest Type 2 cubic graph with girth at least 5 must have at least 34 vertices.

Question 3 Is there a girth $g$ so that all cubic graphs with girth at least $g$ are Type 1? 
An astonishing observation we made when evaluating computational results is that among Type 2 cubic graphs there are very few not containing an induced square.

When testing square-free cubic graphs (but allowing triangles), up to 32 vertices (for 32 vertices alone there are 1.988.135.435.965 connected cubic graphs that are square-free) apart from $K_{4}$ only two graphs - one with 12 vertices and one with 18 vertices - were found that were Type 2 . These graphs are displayed in Figure 7 notice that they have no cycle of length 4 at all.

They can be downloaded from the graph database HoG [6] by searching for the keyword

Type2_quad_free. Allowing squares and forbidding triangles, there are already more than 4.000 .000 Type 2 graphs on up to 26 vertices.
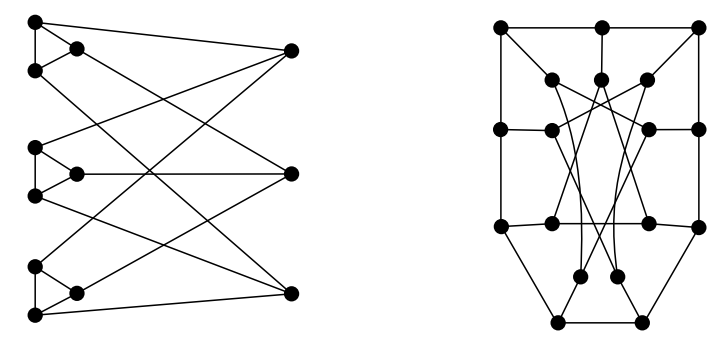

Fig. 7: The only two Type 2 square-free cubic graphs of order up to 32 . The first one is the graph obtained from $K_{3,3}$ by replacing all vertices of one set of the bipartition by triangles, and the second one is the generalized Petersen graph $P(9,3)$.

\section{Acknowledgments}

This research was conducted using the resources of the Stevin supercomputer Infrastructure at Ghent University. Without these resources some results could not have been obtained.

\section{References}

[1] M. Behzad, G. Chartrand, and J. K. Cooper Jr. The colour numbers of complete graphs. J. Lond. Math. Soc., 42:226-228, 1967.

[2] D. Blanuša. Problem cetiriju boja (in russian). Glasnik Mat. Fiz. Astr. Ser. II, 1:31-42, 1946.

[3] G. Brinkmann. Fast generation of cubic graphs. J. Graph Theory, 23(2):139-149, 1996.

[4] G. Brinkmann, J. Goedgebeur, and B. McKay. Generation of cubic graphs. Discrete Math. Theor. Comput. Sci., 13(2):69-79, 2011.

[5] G. Brinkmann, J. Goedgebeur, J. Hägglund, and K. Markström. Generation and properties of snarks. J. Comb. Theory B, 103:468-488, 2013.

[6] G. Brinkmann, J. Goedgebeur, H. Mélot, and K. Coolsaet. House of graphs: a database of interesting graphs. Discrete Appl. Math., 161:311-314, 2013. http://hog.grinvin.org. 
[7] G. Brinkmann, M. Preissmann, and D. Sasaki. Construction of snarks with total chromatic number 5. Technical Report 203, Cahier Leibniz, Laboratoire G-SCOP, INPG, Grenoble, France, 2013.

[8] C. N. Campos, S. Dantas, and C. P. Mello. The total-chromatic number of some families of snarks. Discrete Math., 311:984-988, 2011.

[9] A. Cavicchioli, T. E. Murgolo, B. Ruini, and F. Spaggiari. Special classes of snarks. Acta Appl. Math., 76:57-88, 2003.

[10] U. A. Celmins. A study of three conjectures on an infinite family of snarks. Technical Report 79-19, Dpt. of Combin. Opt., University of Waterloo, Ontario, Canada, 1979.

[11] S. Dantas, C. M. H. de Figueiredo, M. Preissmann, and D. Sasaki. The hunting of a snark with total chromatic number 5. Discrete Appl. Math., 164:470-481, 2014.

[12] B. Descartes. Network-colourings. Math. Gazette, 32:67-69, 1948.

[13] S. Fiorini and R. J. Wilson. Edge-colourings of graphs. Res. Notes Math., 16:49, 1977.

[14] J. L. Fouquet. Note sur la non existence d'un snark d'ordre 16. Discrete Math., 38:163-171, 1982.

[15] D. R. Fulkerson. Blocking and anti-blocking pairs of polyedra. Math. Prog., 1:168-194, 1971.

[16] M. Gardner. Mathematical games: Snarks, Boojums and other conjectures related to the four-colormap theorem. Sci. Am., 234 No. 4:126-130, 1976.

[17] M. K. Goldberg. Construction of class 2 graphs with maximum vertex degree 3. J. Comb. Theory Ser. B, 31(3):282-291, 1981.

[18] G. M. Hamilton and A. J. W. Hilton. Graphs of maximum degree 3 and order at most 16 which are critical with respect to the total chromatic number. J. Combin. Math. Combin. Comput., 10:129-149, 1991.

[19] R. Isaacs. Infinite families of nontrivial trivalent graphs which are not Tait colorable. Amer. Math. Monthly, 82(3):221-239, Mar. 1975.

[20] R. Isaacs. Loupekhine's snarks: a bifamily of non-Tait-colorable graphs. Technical Report 263, Dpt. of Math. Sci., The Johns Hopkins University, Maryland, U.S.A., 1976.

[21] M. Preissmann. Snarks of order 18. Discrete Math., 42:125-126, 1982.

[22] M. Preissmann. C-minimal snarks. Ann. Discrete Math., 17:559-565, 1983.

[23] M. Rosenfeld. On the total chromatic number of a graph. Israel J. Math., 9:396-402, 1971.

[24] P. D. Seymour. Disjoint paths in graphs. Discrete Math., 29:293-309, 1980.

[25] E. Steffen. Non-bicritical critical snarks. Graphs Combin., 15:473-480, 1999.

[26] G. Szekeres. Polyhedral decompositions of cubic graphs. Bull. Austral. Math. Soc., 8:367-387, 1973. 
[27] P. G. Tait. Note on the coloring of maps. Proc. R. Soc., 10:727-728, 1880.

[28] W. T. Tutte. A contribution to the theory of chromatic polynomials. Can. J. Math., 6:80-91, 1954.

[29] V. G. Vizing. On an estimate of the chromatic class of a p-graph. Metody Diskret. Analiz., 3:25-30, 1964.

[30] C. Q. Zhang. Circuit double cover of graphs. London Math. Soc. Lecture Note Ser., 399:253, 2012. 